\title{
Obscured quasars at high redshift in the UKIDSS Ultra Deep Survey
}

\author{
Ismael Botti ${ }^{1}$, Omar Almaini ${ }^{1}$, Will Hartley ${ }^{2}$, \\ Alice Mortlock ${ }^{3}$ and Paulina Lira ${ }^{4}$ \\ ${ }^{1}$ University of Nottingham, School of Physics \& Astronomy, Nottingham, NG7 2RD, U.K. \\ email: ppxib1@nottingham.ac.uk, omar.almaini@nottingham.ac.uk \\ ${ }^{2}$ ETH Zurich, Switzerland \\ email: hartleyw@phys.ethz.ch \\ ${ }^{3}$ Institute for Astronomy, University of Edinburgh, Royal Observatory, Edinburgh, EH9 3HJ, \\ U.K. \\ email: alicem@roe.ac.uk \\ ${ }^{4}$ Depto. de Astronomía, Universidad de Chile, Camino El Observatorio 1515, Santiago, Chile \\ email: plira@das.uchile.cl
}

\begin{abstract}
Obscured quasars hidden in deep X-ray surveys can be recovered by looking at mid-infrared wavelengths, where dust re-radiates the absorbed radiation. Here we present a sample of obscured quasars in the redshift range $1<z<4$ based on data from the UKIDSS Ultra-Deep Survey (UDS), the deepest near-IR survey over $\sim 1$ sq. deg. to date. Candidates that are primarily selected by their $24 \mu \mathrm{m}$ emission are probed by decomposing their spectral energy distribution (SED) to disentangle the emission from the AGN and its host galaxy. We show preliminary results on their host galaxy properties as well as their clustering, showing that obscured quasars are found in galaxies located in the green valley, residing in dark matter haloes not different from normal galaxies at those redshifts.
\end{abstract}

Keywords. galaxies: evolution, galaxies: high-redshift, quasars: general, infrared: galaxies

\section{A hidden population of active galactic nuclei}

Supermassive black holes at the centre of galaxies have shown to be crucial in the galaxy formation and evolution paradigm. For more than a decade, it has been known that black hole masses correlates with several host galaxy spheroid properties at different scales such as stellar mass, stellar velocity dispersion and luminosity (Marconi \& Hunt 2003, Magorrian et al. 1998, Ferrarese \& Merritt 2000, Gebhardt et al. 2000, Ferrarese 2002) suggesting that the grow of the both are intimately connected (Silk \& Rees 1998, Di Mateo et al. 2005). Therefore a complete census of these supermassive black holes is required in order to understand the formation and evolution of galaxies. This is even more crucial when probing the high redshift (high- $z$ ) universe, when most of the growth of the stellar and black hole mass occurred.

Active galactic nuclei (AGN) are efficiently found by their X-ray emission. The hard Cosmic X-ray Background spectrum (Gendreau et al. 1995), however, revealed a hidden population that is so heavily obscured that much of it is missed even in the deepest X-ray surveys to date (Gilli et al. 2001, Comastri et al. 2001). These missing objects can be recovered in the mid-infrared, where strong emission from hot dust in the surroundings of the central engine can be detected. 
In this work we present preliminary results of a search for a large sample of obscured AGN at high redshifts based on their mid-infrared emission and spectral energy distribution (SED) shape.

\section{The UKIDSS Ultra-Deep Survey}

Deep surveys are crucial to obtain a large sample of obscured quasars to unveil their properties statistically. We used data from the eighth data release (DR8) of the UKIDSS Ultra-Deep Survey (UDS), the deepest near-infrared survey over $\sim 1$ sq. deg. to date. The UDS has a rich multiwavelength coverage from X-rays to radio, and is particularly deep at optical, near-IR and mid-IR wavelengths, making of it a unique dataset for studying the evolution of distant galaxies. Indeed, the UDS is so deep $(K=24.6, H=24.2$ and $J=24.9,2^{\prime \prime}$ AB magnitudes, $5 \sigma$ ) that we are able to probe typical sub- $L^{*}$ galaxies in an un-biased manner to $z \sim 3$ Cirasuolo et al. 2010, and the huge number of galaxies detected up to $z \sim 6$ enable us to trace the dark matter distribution to study the large scale structure.

\section{Obscured quasars in the UDS}

Obscuration in AGN comes from large gas column densities, associated with large amount of dust which absorbs rest frame UV-optical radiation and re-radiates in the infrared (IR). Therefore, we would expect obscured quasars to be bright at mid-IR wavelengths due to hot dust emission, which is probed at high- $z$ by the observed $24 \mu \mathrm{m}$ flux (rest frame $5-12 \mu \mathrm{m}$ ).

We selected all $24 \mu \mathrm{m}$ detected sources in the redshift range $1<z<4$ as AGN candidates. However, the high luminosity imposed by the flux cut $\left(f_{\nu}(24 \mu \mathrm{m}) \geqslant 300 \mu \mathrm{Jy}\right)$ and redshift range imply that candidates are likely to be powerful star forming galaxies with strong PAH emission or quasars with strong dust emission from the central region. To disentangle the relative contribution of both, host and quasar, we followed an SED decomposition approach (Bongiorno et al. 2012, Lusso et al. 2011) which consists on fitting the available photometry to a composite SED model given by a linear combination of host galaxy and AGN emission. The host galaxy is modelled as a linear combination of templates from Bruzual \& Charlot (2003; stellar emission) and Mullaney et al. (2011; PAH emission), and the template of Richards et al. (2006) was used for the AGN component. We allowed for independent dust extinction for each component, using the prescription from Calzetti et al. (2000) for the host and a SMC-like extinction law for the AGN component given by Prevot et al. (1984). The best fit was found by using a $\chi^{2}$ minimisation algorithm.

Our sample is composed by 323 objects with $A_{V} \geqslant 4$, equivalent to $E(B-V) \geqslant 1.4$, and dominated by AGN emission, whose SED contribution is larger than 60 per cent between $1-10 \mu \mathrm{m}$. We have also quantified the number of false detections using simulations, which is lower than 10 per cent.

\section{Host galaxy properties and clustering}

The high extinction of the central engine in these objects allows the study of the host galaxy because optical and near-IR wavelengths are dominated by stellar emission. From SED fitting we obtained stellar masses and rest frame magnitudes for the hosts, enabling a direct comparison with normal galaxies in the survey with the same sample characteristics (mass and redshift distribution). In figure 1 we show the mass and rest 

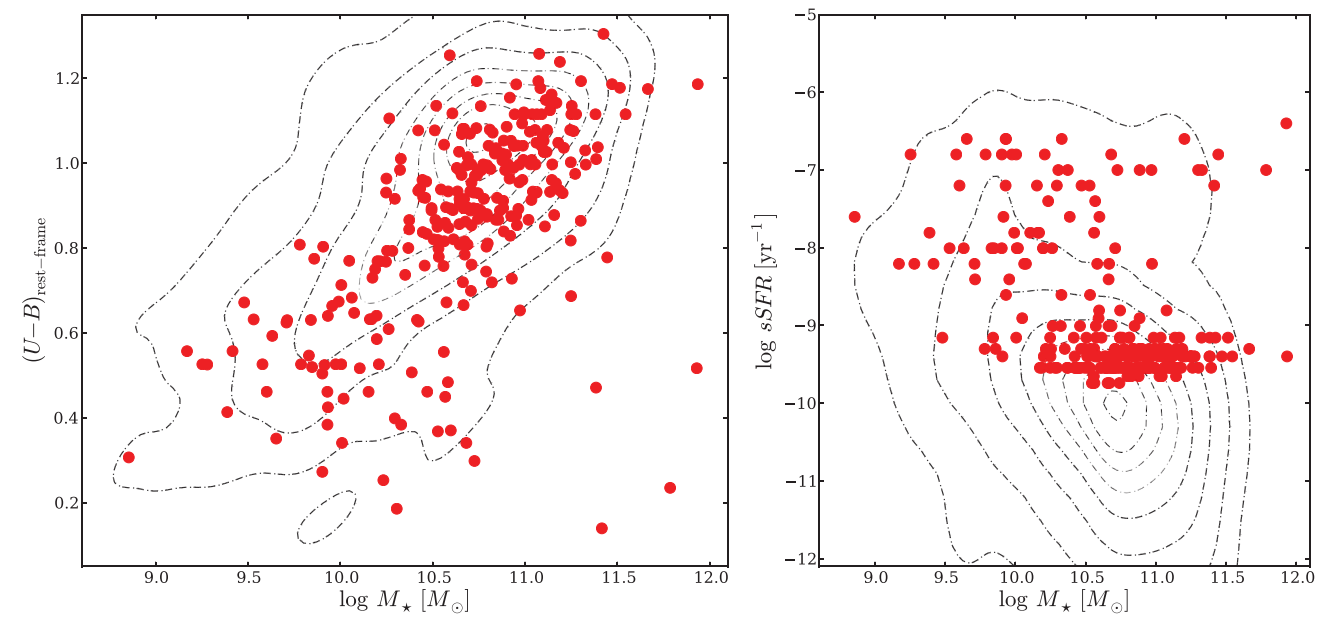

Figure 1. Left: Rest frame $U-B$ colour against stellar mass of the obscured quasar hosts (points). Contours show the distribution of the control sample of normal galaxies. Right: Specific star formation rate $(s S F R)$ as a function of the host stellar mass. Control sample is shown as contours.

frame $U-B$ colour of our sample in comparison with a control sample. Our sample's hosts tend to lie in the most massive end of the distribution, with more than the 80 per cent of the sample with $M \geqslant 10^{10.3} M_{\odot}$, and showing red $U-B$ colour. With the help of the UVJ criteria (Williams et al. 2009, Hartley et al. 2013) and specific star formation rates $\left(s S F R=S F R / M_{\star}\right)$ from SED fitting we concluded that the bulk of the hosts correspond to galaxies in the so called green valley, with relatively low specific star formation rates but not too low to be considered quiescent galaxies (see figure 1).

In order to probe the typical dark matter halo masses in which our sample reside we have performed a clustering analysis using galaxies in the UDS field as tracers of the underlying dark matter distribution. The procedure, described in detail in Hartley et al. (2013), was applied in two wide redshift range $1.1<z<2$ and $2<z<3.3$, probing a comoving distance of $\sim 1500 \mathrm{Mpc}$ each. Preliminary results show that our sample is found to be in dark matter halo masses of the order of $\sim 5 \times 10^{12} M_{\odot}$, in between those where typical star forming and quiescent galaxies reside (Hartley et al. 2013, see figure 2), and not significantly different from the halo mass obtained for the general galaxy population at those redshifts. A more detailed study of the clustering as a function of AGN (luminosity) and host properties (luminosity, colours, etc) is underway.

\section{Conclusions}

We have presented a sample of obscured quasars and properties of their host galaxies and clustering in the UKIDSS Ultra-Deep Survey. We have shown that host galaxies are mostly star forming with red $U-B$ colours, suggesting large amount of dust available within the hosts. Clustering analysis have shown that our sample reside in average dark matter haloes at those redshifts.

So far, our results can only be taken as preliminary, as we are improving the selection technique by using robust SED fitting methods (e.g. using Markov Chain Monte Carlo algorithms, see Calistro Rivera et al., this proceeding), that will be presented soon in Botti et al. (in prep.). 


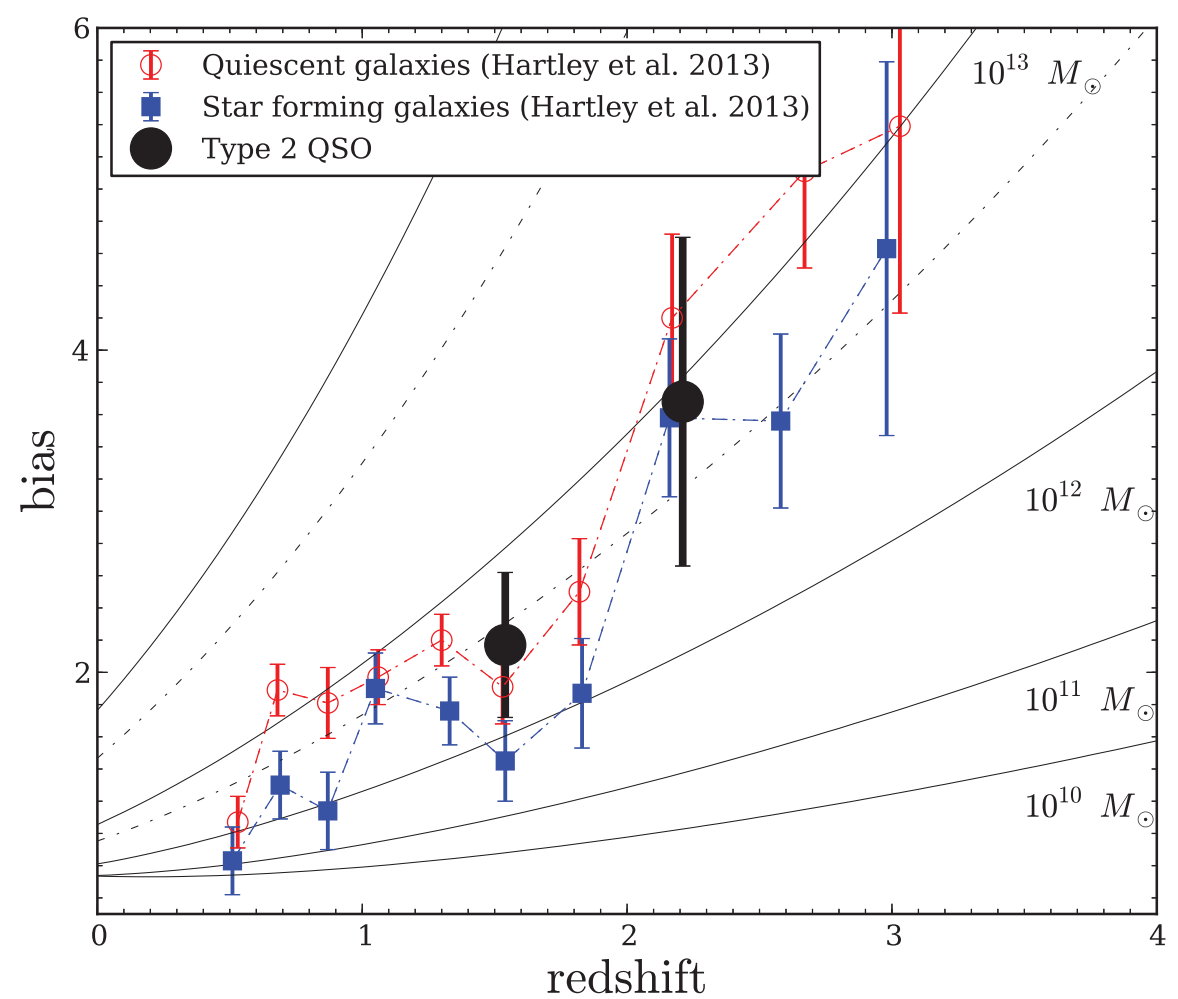

Figure 2. Bias inferred from cross-correlation analysis against redshift for the obscured quasar sample (points). Passive and star-forming galaxy biases from Hartley et al. (2013) are shown as the upper and lower dash-dotted curves respectively. Solid and dashed lines show the bias for dark matter halos of various masses (in solar masses, as labelled).

\section{References}

Bongiorno A., Lusso E., Comastri A., Vignali C., et al. 2012, MNRAS, 427, 3103

Bruzual A. G. \& Charlot S. 2003, MNRAS, 344, 1000

Calzetti D., Armus L., Bhlin R. C., et al. 2000, ApJ, 533, 682

Cirasuolo M., McLure R. J., Dunlop J. S., et al. 2010, MNRAS, 401, 1166

Comastri, F. Fiore, C. Vignali, et al. 2001, MNRAS 327, 781

Di Matteo, T., Springel V. \& Hernquist L. 2005, Nature, 433, 604

Ferrarese L. 2002, ApJ, 578, 90

Ferrarese L. \& Merritt D. 2000, ApJ, 539, 9

Gebhardt K., Bender R., Bower G., et al. 2000, ApJ, 539, L13

Gendreau K., Mushotzky R., Fabian A. et al. 1995, PASJ, 47, L5

Gilli R, Salvati M., Hasinger G. 2001, A\&A 366, 407

Hartley W. G., Almaini O., Mortlock A., et al. 2013, MNRAS, 431, 304

Lusso E., Comastri A., Vignali C., et al. 2011, A\&SA, 534, 110

Magorrian J. et al. 1998, AJ, 115, 2285

Marconi A. \& Hunt L. K. 2003, ApJL, 589, 21

Mullaney J. R., Alexander D. M., Goulding A. D., et al. 2011, MNRAS, 414, 1082

Prévot M. L., Lequeux J., Maurice E., et al. 1984, A\&A A, 132, 389

Richards G., Lacy M., Storrie-Lombardi L., et al. 2006, ApJS, 166, 470

Silk J. \& Rees M. J. 1998, A\&SA, 331, 1

Tremaine S., Gebhardt K., Bender R., et al. 2002, ApJ, 574, 740

Williams R. J., Quadri R. F., Franx M., et al. 2009, ApJ, 691, 1879 